Supporting Information

\title{
Hydrogel Pressure Distribution Sensor Based on Imaging Strategy and Machine Learning
}

\author{
Zhengxin Liu${ }^{1 \#}$, Tingwei Zhang ${ }^{1 \#}$, Mei Yang ${ }^{1}$, Weizheng Gao1, Songjie $W^{2}{ }^{2}$, \\ Kaile Wang ${ }^{1}$, Feihong Dong ${ }^{1}$, Jie Dang ${ }^{1}$, Diange Zhou $^{3^{*}}$ and Jue Zhang ${ }^{1,2^{*}}$ \\ 1 Academy for Advanced Interdisciplinary Studies, Peking University, Beijing 100871, People's Republic \\ of China \\ 2 College of Engineering, Peking University, Beijing 100871, People's Republic of China \\ 3 Arthritis Clinic \& Research Center, Peking University People's Hospital, Beijing 100044, People's \\ Republic of China \\ \#: These authors contributed equally to this work.
}

* Corresponding authors: Diange Zhou (Email: zhoudiange@126.com) and Jue Zhang (Email: zhangjue@pku.edu.cn ) 


\section{SEM and XPS Characterization of Hydrogels}

To investigate the morphology of PAAm/PAA hydrogels and PAAm/PAA-Fe ${ }^{3+}$ hydrogels, a scanning electron microscope (SEM, S-4800, Hitachi, Japan) at $1 \mathrm{kV}$ without sputtering gold particles was used. Samples were freeze-dried with a freeze dryer (Christ Alpha 2-4 LDplus, Martin Christ, Germany) before testing.

X-ray photoelectron spectroscopy (XPS) was used to examine the surface element species and valence states of the PAAm/PAA hydrogels and PAAm/PAA-Fe ${ }^{3+}$ hydrogels to investigate whether the bonds of second network were formed. Samples were freezedried with a freeze dryer before testing. An Al monochromatic X-ray excitation source with an excitation energy of $149.94 \mathrm{~W}$ was used.

\section{Circuit Design, Signal Acquisition and Data Analysis of Self-designed EIT System}

Figure $\mathrm{S} 1$ indicates the block diagram of a self-designed EIT system hardware, including excitation signal chain, measuring signal chain, and control unit. The excitation signal chain was primarily designed to generate an excitation current with controllable frequency and amplitude. For this, a direct digital synthesis (DDS) chip is adopted to generate a sinusoidal voltage signal $\mathrm{V}(\mathrm{f})$ with programmable frequency. Then, a variable gain amplifier was used to adjust the amplitude of $V(f)$ and get $V(a, f)$. Subsequently, the $V(a, f)$ was fed into a Howland current source to generate the single-end excitation current signal $C(a, f)$. Two 16:1 multiplexers were controlled to route the current to a pair of excitation electrodes. 
At this point, the current flows through the hydrogel to the ground, producing measurable voltage between the measuring electrodes. In the measuring signal chain, two 16:1 multiplexers were also used to select a pair of measuring electrodes and connect them to the differential input of the instrumentation amplifier (INA). The INA amplifies the voltage signal with a fixed gain and output it to the bandpass filter, which is used to suppress noise. Then, a variable gain amplifier is also equipped to get a suitable voltage range for $A D C$ sampling.

The ADC is built in the micro control unit (MCU). As shown in Figure S1, all components indicated by the dotted boxes can be adjusted by the MCU. Especially, the multiplexer switching is controlled by a common EIT drive pattern: adjacent drive pattern. There are 16 different current excitation routes and each arrangement has 16 differential voltage measurements (i.e., 13 measurements and 3 zeros). After each switching and sampling, the root mean square value of the sampled sinusoidal voltage signal is calculated by MCU as an effective measurement value. So, $16^{*} 16=256$ measurements are collected and sent to the computer via USB port for one image reconstruction.

\section{FCN Network Architecture}

A fully connected neural network (FCN) with two hidden layers and input and output layers was constructed (as shown in Figure S3). The activation function of hidden layer was ReLU. In order to improve the generalization ability of the FCN model and prevent over-fitting, the dropout layer (dropout ratio is 0.2 ) was set before the output layer. Mean 
Squared Error (MSE) loss was chosen as the loss function, the optimizer is Adam, and the learning rate was 0.01 . Batch-size $=128$, epoch $=10000$. 


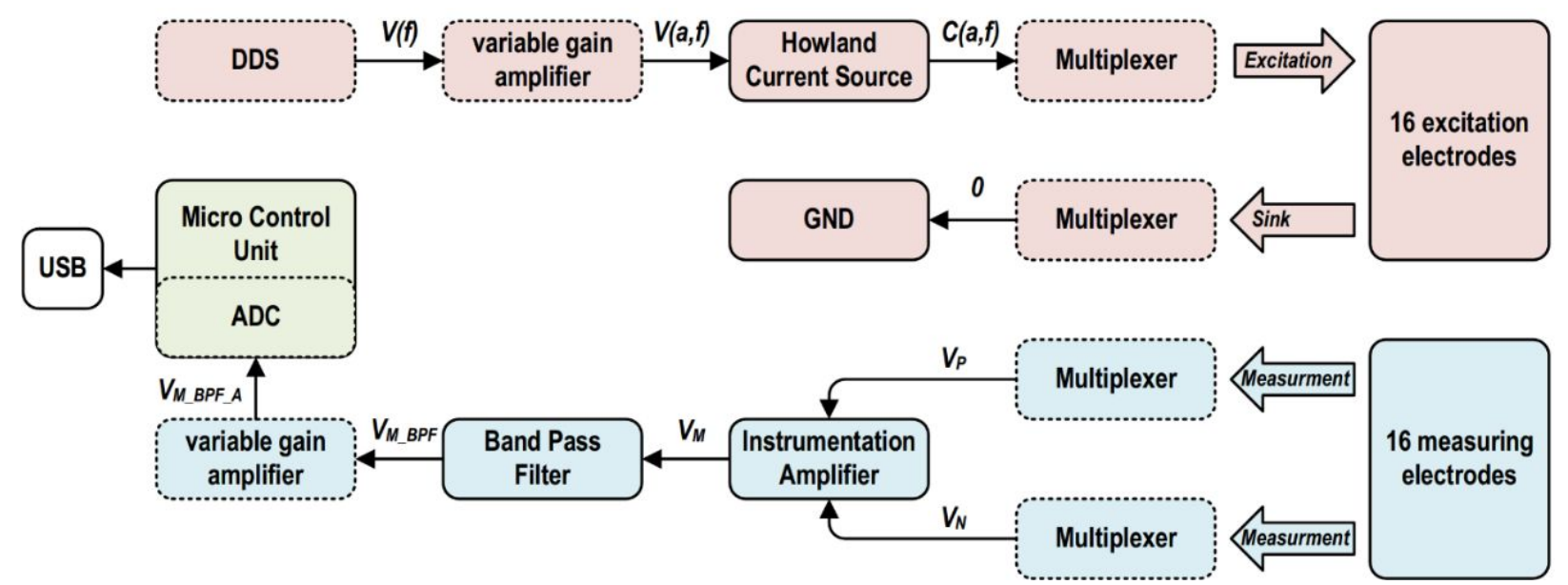

Figure S1. The block diagram of self-designed 16 electrodes EIT system hardware. The system consists of an excitation signal chain (red boxes), measuring signal chain (blue boxes) and control unit (green boxes). The arrows represent the flow direction of signal. The solid line boxes indicate unadjustable components, and the dotted boxes indicate components with variable parameters that can be adjusted by control unit. 


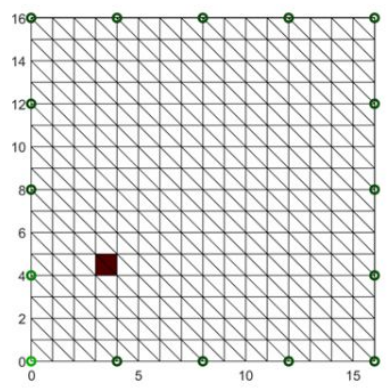

(a) 4352

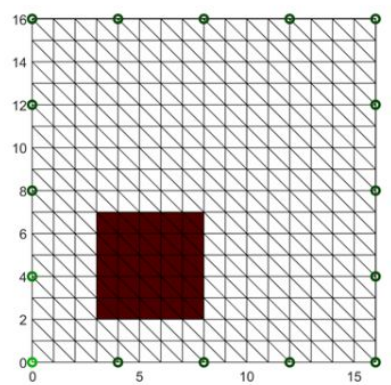

(b) 19532

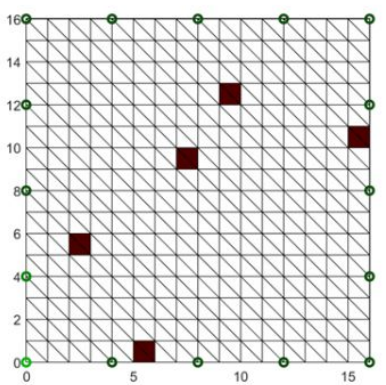

(c) 50373

Figure S2. Typical distribution and quantity of different samples; (a) one single small square area is compressed; (b) a large square area (greater than or equal to $2 \times 2$ ) is compressed; (c) multiple independent small square areas are compressed. 


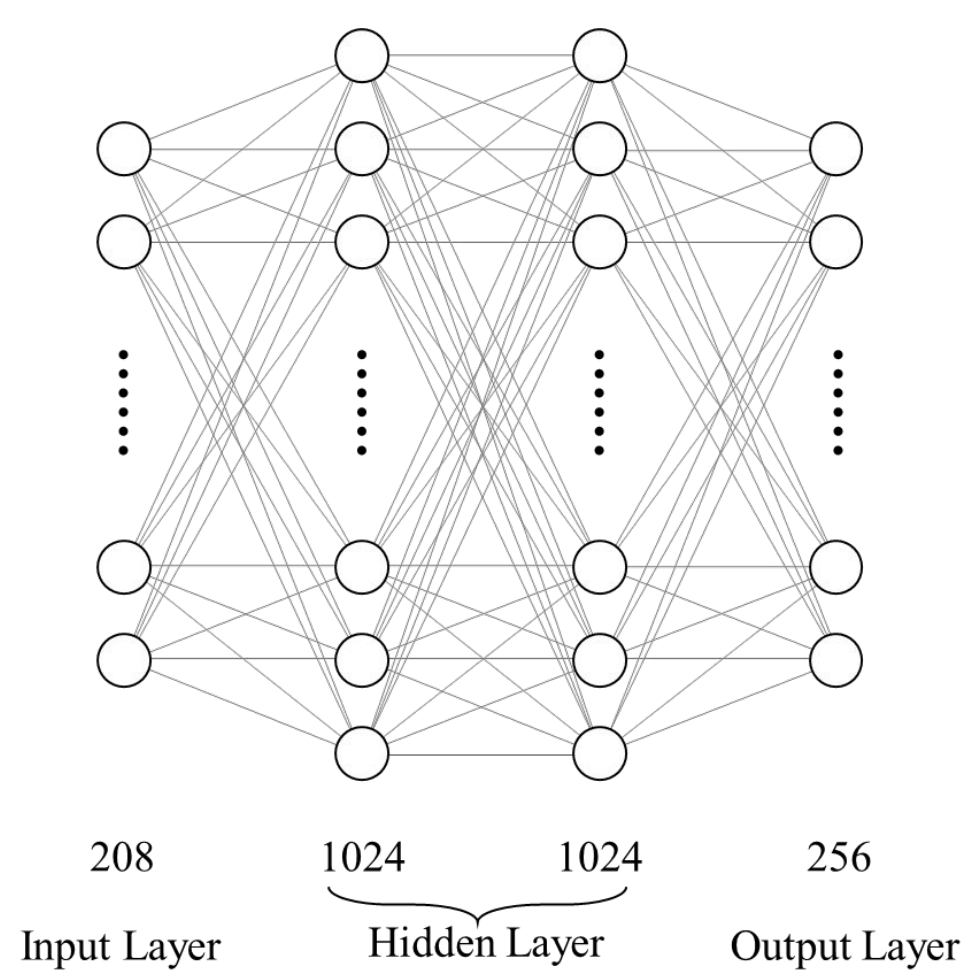

Figure S3. Structure of the FCN 


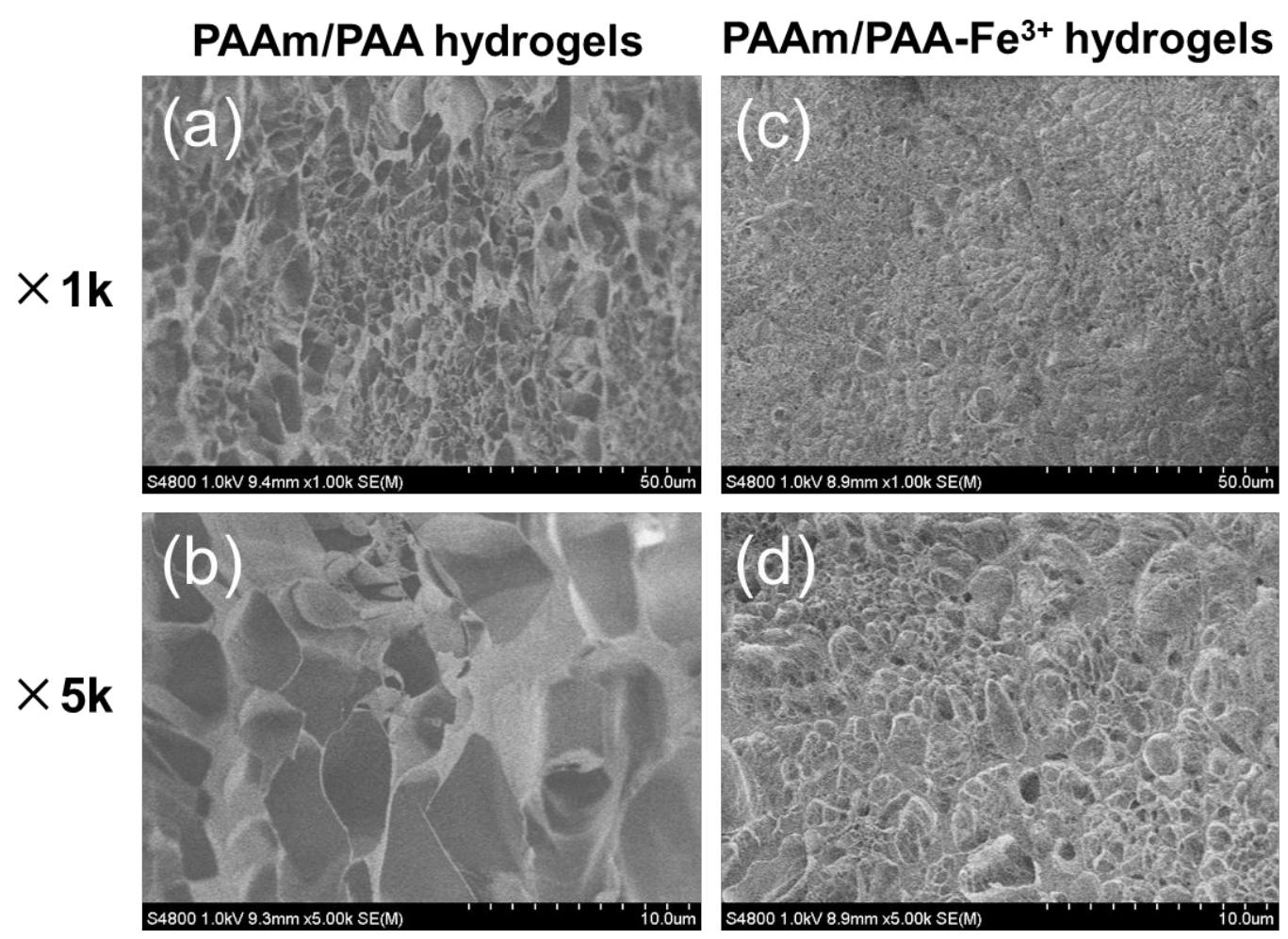

Figure S4. SEM micrographs of different hydrogels: $(a, b)$ control group PAAm /PAA hydrogel; (c, d) PAAm/PAA-Fe ${ }^{3+}$ hydrogels (DN hydrogels); (a, c) SEM micrographs with a magnification of $1 \mathrm{k} ;(b, d)$ SEM micrographs with a magnification of $5 \mathrm{k}$. 

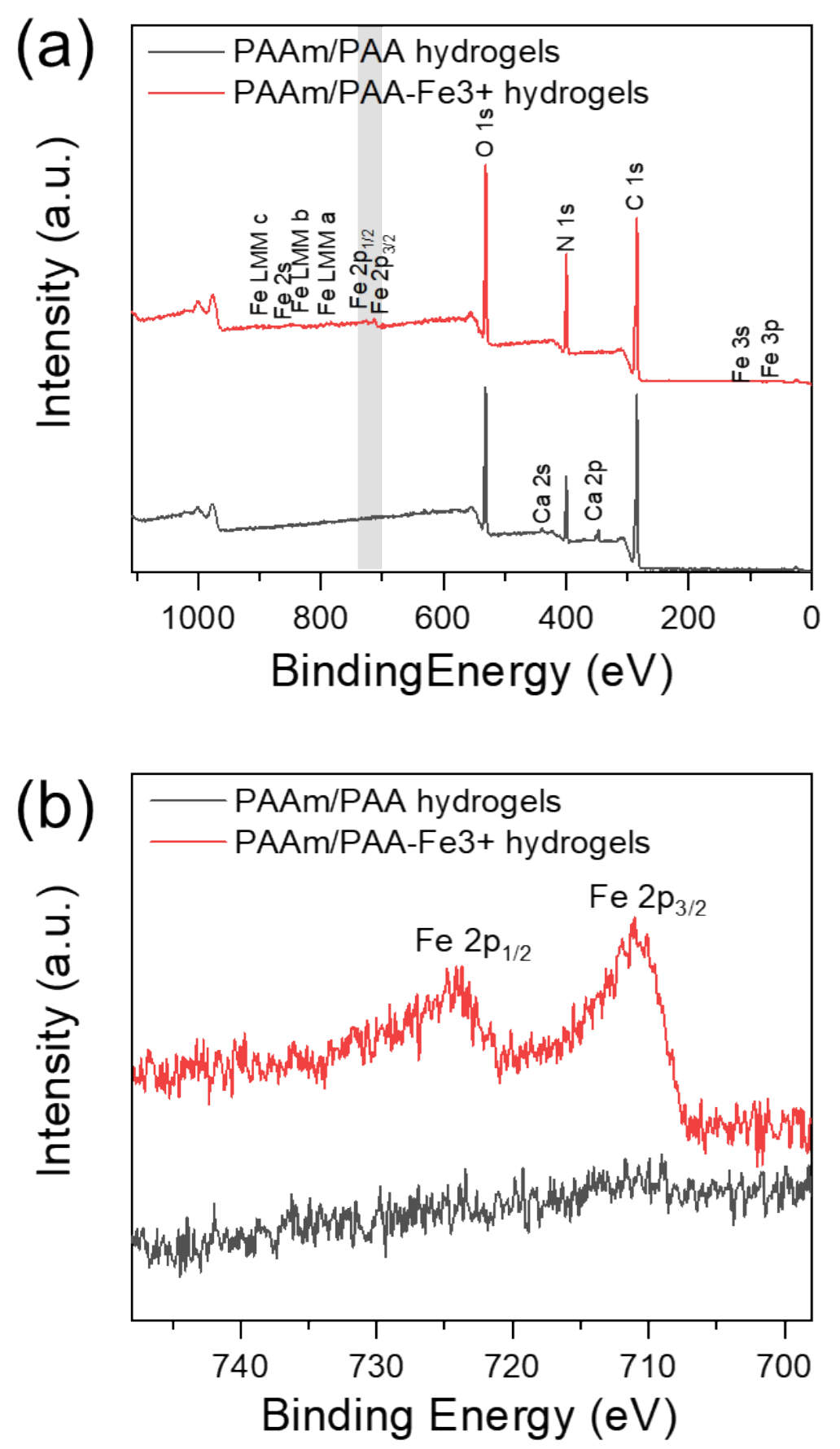

Figure S5. XPS spectrum of PAAm/PAA hydrogels (control group) and PAAm/PAA-Fe ${ }^{3+}$ hydrogels (DN hydrogels): (a) full spectrum; (b) high resolution spectrum of Fe $2 p$ 

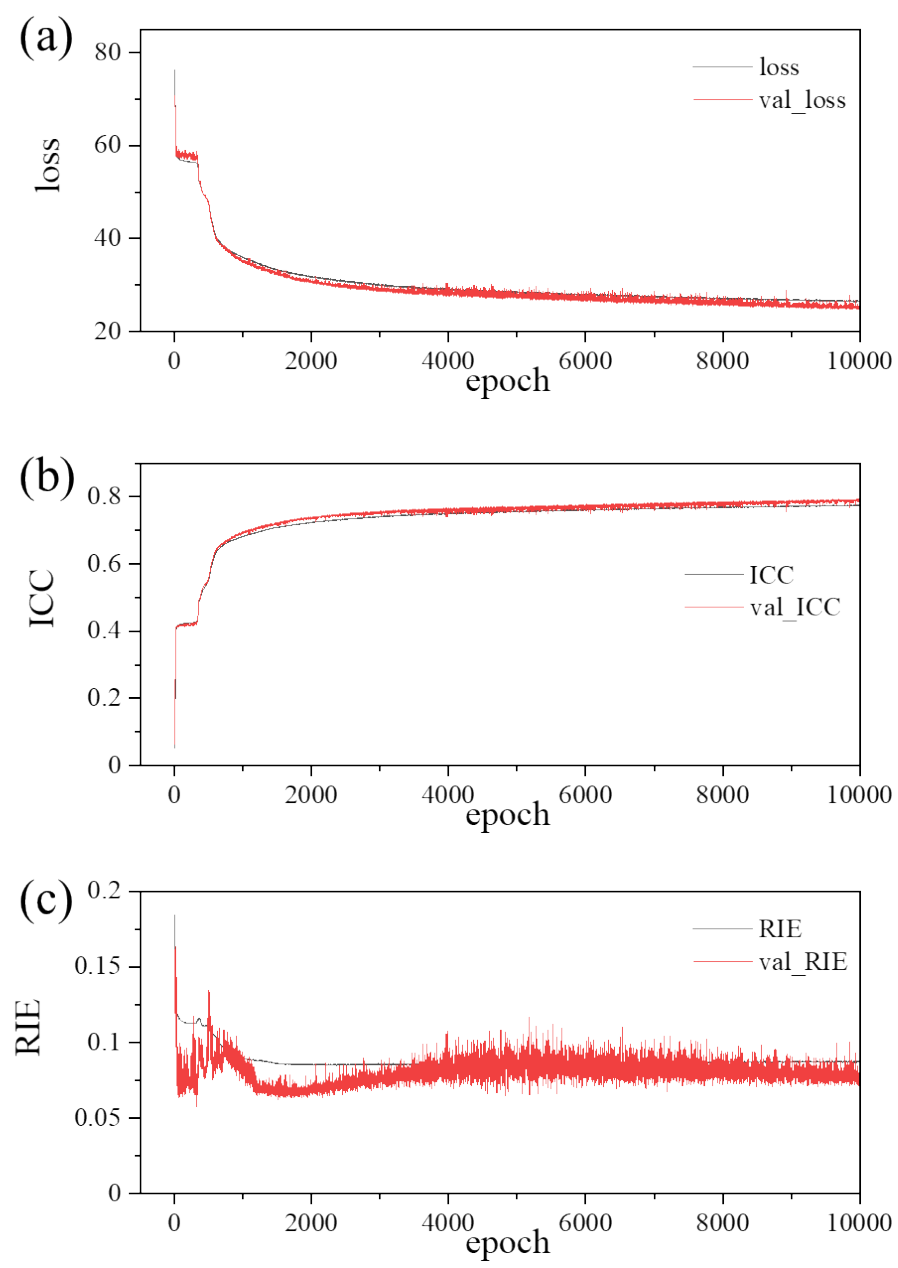

Figure S6. The relationship between the evaluation parameters of the FCN model and epoch: (a) the loss curve of FCN; (b) the ICC curve of FCN; (c) the RIE curve of FCN. 
Table S1 The MSE loss values, and the RIE and ICC values of the 10 -fold cross-validation

\begin{tabular}{ccccc}
\hline & Average Value & Standard Deviation & Max & Min \\
\hline MSE Loss & 25.020 & 0.899 & 26.717 & 24.047 \\
ICC & 0.733 & 0.011 & 0.746 & 0.712 \\
RIE & 0.078 & 0.004 & 0.088 & 0.073 \\
\hline
\end{tabular}

